\title{
On the Impact of Coverage Range on AIS Message Reception at Flying Platforms
}

\author{
Federico Clazzer $^{\ddagger}$, Andrea Munari ${ }^{\ddagger}$, Simon Plass ${ }^{\ddagger}$ and Birgit Suhr* \\ $\ddagger$ DLR - German Aerospace Center, Institute of Communications and Navigation - Wessling, Germany \\ * DLR - German Aerospace Center, Institute of Space Systems - Bremen, Germany \\ \{federico.clazzer, andrea.munari, simon.plass, birgit.suhr\}@dlr.de
}

\begin{abstract}
In the recent past, an increasing interest has been devoted to the possibility of receiving Automatic Identification System (AIS) messages via Low Earth Orbit (LEO) satellites. While the principle has been demonstrated to be a viable option for monitoring vessel traffic over oceans and vaste land areas, the achievable performance from a communications viewpoint is far from optimal. Recently, it was shown how AIS traffic seen at a satellite can be very accurately modeled resorting to simple random access schemes. Leveraging this result, in this work we propose a simple yet flexible analytical framework capable of predicting channel load and overall reception performance taking into account the spatial distribution of vessels as well as their traffic generation pattern. Feeding the model with ship speed and location data derived from experimental settings, we discuss the achievable efficiency for a typical LEO-satellite detecting AIS packets. Moreover, the impact of the receiver footprint on ground on the overall decoding performance is investigated, deriving some interesting insights on the benefits that could stem resorting to narrower-beam systems. In this direction, we discuss two cases: the usage of a LEO satellite with a directional antenna soon to be launched for AIS monitoring, and the possibility of using airliner for receiving vessel-generated traffic.
\end{abstract}

\section{INTRODUCTION}

In the last years, satellite-aided Automatic Identification System (AIS) has become one of the hot topics in the maritime safety and security field, drawing the attention of both researchers and standardization bodies [1]. The AIS standard [2], developed in the 1990's and now a mandatory feature for commercial vessels in most countries, dictates boats to periodically broadcast in the VHF band location and speed information, which can be used by surrounding vessels and by operators in the mainland to prevent collisions and make local traffic decisions.

A major breakthrough in the field was achieved in the mid 2000 's, when several studies proved the viability of AIS message reception at Low Earth Orbit (LEO) satellites [3], [4], [5], [6], [7]. As a matter of fact, the possibility to collect navigation information at a flying platform complements the original goal of AIS, providing an overall picture of vessels distributed over large areas and enabling worldwide routes monitoring. This, in turn paved the way to an unexplored plurality of commercial applications which are today an expanding reality, ranging from goods and ship tracking to oceans monitoring for both environmental and safety purposes.

Despite the increasing attention devoted to research and development in the field, reception of AIS messages at a satellite is in general not yet efficient from a communications perspective. The standard, in fact, was originally devised to enable reliable data exchanges among a relatively small population of vessels, and resorts to a medium access scheme which distributedly forms clusters where messages are sent in a coordinated fashion. On the other hand, when a satellite footprint covering areas of several thousands of square kilometers is considered, the contribution of many clusters not in visibility of each other - and thus not coordinated - will result in packet collisions at the receiver, with detrimental effects on decoding performance. Especially in densely ship populated regions this issue affects dramatically the tracking performance of a satellite-aided AIS system, all the more so considering the steadily increasing traffic generated by other maritime communication services being allocated to VHF band [8].

An interesting characterization of the problem was given in [9], where the authors showed by means of a simple analytical framework how incoming AIS traffic at a Low Earth Orbit (LEO) satellite can be very accurately modeled considering a slotted Aloha access scheme that disregards any form of coordination among vessels. Taking the lead from this, and relying on experimental data for ships distribution, it was possible to prove how the average load to be expected in regions of interest such as the Mediterranean see or the western coasts of Europe can easily be very high (e.g, larger than 5 $\mathrm{pk} / \mathrm{slot}$ ). Therefore, although some recent works have tried to improve decoding capabilities at the satellite resorting to advanced signal processing techniques [10], it is clear that a limiting factor to the overall achievable performance is given by the footprint used to collect AIS messages. In this perspective, an interesting and key tradeoff arises: on the one hand a larger coverage radius is desirable, so to get a snapshot on a wider area; on the other hand, the broader the footprint, the higher the traffic and the lower the decoding probability.

Within this paper, we study this tradeoff in greater detail, deriving some insights of interest both from a research and a practical viewpoint. Extending the work in [9], we develop an analytical model to characterize relevant metrics such as throughput and detection probability. To achieve this we work at packet level, considering collisions among messages as destructive and abstracting physical layer details. While such an approach is particularly useful to derive closedform expressions that capture the key parameters coming into play, it also provides a reasonable first approximation of the performance proper of the rather simple physical layer of AIS. The framework we introduce is rather flexible, and can take as input any geographical density for vessels as well as any velocity profile of interest. Within this work, in particular, 
we present results obtained considering experimental data for both aspects, and we elaborate on the decoding behavior at a flying platform referring to three cases of practical relevance, encompassing a typical LEO satellite with coverage radius of approximately $2500 \mathrm{~km}$; a satellite developed by the German Aerospace Center (DLR) for launch in 2014 with a reduced footprint; and a scheduled commercial aircraft, i.e., airliner, flying over an area of interest.

We start our discussion in Section II by providing a short description of some relevant aspects of the AIS communication protocols. Then, Section III introduces an analytical framework to evaluate under certain assumptions the load and throughput achievable when decoding AIS packets at a flying object characterized by a specific coverage range. The model is subsequently used in Section IV to evaluate worldwide the performance tradeoff induced by the reception radius under realistic ship distribution and to identify possible optimization schemes. The conclusions of our work are finally drawn in Section V.

\section{Automatic Identification System (AIS)}

AIS was designed in the 90's as a system for automatic vessel identification in order to improve safety of navigation and increase the level of sea surveillance. While a comprehensive description of the standard goes beyond the scope of this work, we briefly report in this section an overview of some relevant aspects of the communication protocol which are relevant for the following discussion.

At the physical layer, two VHF channels around $160 \mathrm{MHz}$ (161.975 MHz and $162.025 \mathrm{MHz}$ ) are available, with $9.6 \mathrm{kbit} / \mathrm{s}$ bit rate and Gaussian minimum-shift keying (GMSK) modulation. No Forward Error Correction (FEC) nor interleaving is used [2]. Frames of one-minute duration and composed of 2250 slots repeat over time, and vessels are assumed to be slot-synchronized, e.g., by means of a GPS signal. Within each frame a ship can signal to its neighbors or to the costal authorities relevant information on its status, including position, speed and direction updates by transmitting AIS messages of the duration of one slot. In order to ensure a certain level of reliability for packet delivery, the standard defines at the MAC layer 4 different access schemes depending on the mode of operation. The most used, and taken as reference throughout this paper, is Self-Organized Time Division Multiple Access (SOTDMA), foreseen for vessels traveling along a specific route called continuous operation. SOTDMA is based on a distributed clustering concept in which vessels within each other's coverage range distributively coordinate so to avoid collisions among packets. In particular, each vessel's AIS receiver keeps track of the slot occupation with the help of a dynamic directory of received neighbors, and sends its messages only over slots that are perceived as free. Once a slot is occupied by a ship, it may be booked for a certain number of frames indicated in a time out field of the AIS message, so to ease negotiation procedures.

The frequency with which ships send updates depends on the speed, so to allow proper tracking and collision avoidance capabilities. In particular, AIS defines 4 groups of frequencies that shall be used by vessels. Ships travelling with a speed up to 3 Knot (kn) send AIS packets every $180 \mathrm{~s}$, ships travelling with a speed between $3 \mathrm{kn}$ and $14 \mathrm{kn}$ send AIS packets every
$10 \mathrm{~s}$, ships travelling with a speed between $14 \mathrm{kn}$ and $23 \mathrm{kn}$ send AIS packets every $6 \mathrm{~s}$, while ships travelling with a speed above $23 \mathrm{kn}$ send AIS packets every $2 \mathrm{~s}$.

\section{A CHARACTERIZATION OF AIS TRAFFIC LOAD AT THE SATELLITE}

Throughout our investigation we focus on a flying platform, e.g., a satellite or an airliner, collecting AIS messages transmitted by ships within its coverage range. As discussed in Section II, time is divided in slots, each of them of the duration of one data unit. Reception at the platform is modeled at a packet level, abstracting the underlying physical layer, and collisions are regarded as destructive. According to this assumption, an AIS message sent by a vessel is successfully retrieved only if no other peer accessed the channel over the same slot. An exact characterization of the traffic pattern generated as per SOTDMA by ship clusters falling within the footprint of the receiver and not coordinating among each other is in general not trivial. On the other hand, [9] showed that a very good approximation can be obtained assuming all vessels to simply generate messages according to a Poisson process of aggregate intensity $G$ and accessing the medium as soon as data units are available for transmission. Leveraging this result, we model the flying platform as the receiver in a well-known Slotted ALOHA (SA) protocol.

This working hypothesis is particularly useful, since the performance of the system can be extensively characterized as soon as the channel load $G$, expressed in packets per slot, is available. This parameter, in turn, depends on the number of transmitters and on the message generation rate associated to each of them, and brings the AIS traffic and topology properties into the model. In the case of interest, in fact, the transmitters population coincides with the number of vessels $n$ that fall within the footprint of the receiver, while the transmission frequency $\omega$ (measured in packets per slot) depends on the vessel speed, as defined in the AIS standard [2]. The former parameter can be computed for any vessel spatial distribution $f_{S}(\varphi, \lambda)$ over the Earth surface as:

$$
n=\iint_{(\varphi, \lambda) \in \mathcal{A}} f_{S}(\varphi, \lambda), d \varphi d \lambda .
$$

where $(\varphi, \lambda)$ represent the coordinates of a point in latitude and longitude, and $\mathcal{A}$ is the region on the terrestrial sphere described by the reception pattern of the flying platform. Recalling that the standard foresees four different transmission frequencies $\omega_{i}$ for ships, the overall expected channel load can be written as

$$
G=\mathbb{E}\left[\sum_{i} n_{i} \omega_{i}\right]=\sum_{i} \mathbb{E}\left[n_{i}\right] \omega_{i},
$$

where $n_{i}$ is the number of ships that generate messages with frequency $\omega_{i}$. In turn, $n_{i}$ can readily be expressed as soon as the probability density function (PDF) $f_{v}$ of vessels speed is available:

$$
n_{i}=n \int_{v_{i-1}}^{v_{i}} f_{v}(v) d v
$$


where $\left[v_{i-1} ; v_{i}\right]$ is the velocity range in which AIS messages are sent with frequency $\omega_{i}$.

Combining (1) and (3), we finally obtain:

$$
G=\iint_{(\varphi, \lambda) \in \mathcal{A}} f_{S}(\varphi, \lambda) d \varphi d \lambda \sum_{i}\left[\omega_{i} \int_{v_{i-1}}^{v_{i}} f_{v}(v) d v\right]
$$

which allows to compute the channel load for any geographic distribution and mobility model of interest. On the other hand, (4) also highlights the dependency of $G$ on the footprint, which, as discussed in Section I, triggers a key tradeoff for the overall system performance. $\mathcal{A}$, in fact, corresponds to the portion of the Earth surface that is covered by the receiver when placed in a specific location, which depends in general on the antenna coverage and on the height of the platform.

In order to further elaborate a general framework, let us assume a circular receiver's coverage area of radius $r$. Under this hypothesis, the latitudes spanned by $\mathcal{A}$ can be easily evaluated, as they only depend on $r$. On the contrary, the lower and higher longitude integration endpoints in (4) $\left(\lambda_{\min }\right.$ and $\lambda_{\max }$, respectively) are a function of both latitude and radius $r$ and can be evaluated with the help of the haversine formula. In fact, if the receiver is located at $\left(\varphi_{c}, \lambda_{c}\right)$, any point of coordinates $(\varphi, \lambda)$ on the Earth surface lying on the circumference of the footprint satisfies:

$$
r=2 R_{E} \arcsin (a)
$$

where $R_{E}$ is the Earth radius, and $a$ is given by

$$
a=\sqrt{\sin ^{2}\left(\frac{\varphi-\varphi_{c}}{2}\right)+\cos \left(\varphi_{c}\right) \cos (\varphi) \sin ^{2}\left(\frac{\lambda-\lambda_{c}}{2}\right)}
$$

With few mathematical manipulations, we can find the expression of the sought integration endpoints as the solutions $\lambda_{\min }$ and $\lambda_{\max }$ of (5) as

$$
\begin{gathered}
\lambda_{\min }=\min \left\{\lambda_{c}+\arccos (b), \lambda_{c}-\arccos (b)\right\} \\
\lambda_{\max }=\max \left\{\lambda_{c}+\arccos (b), \lambda_{c}-\arccos (b)\right\},
\end{gathered}
$$

where $b$ is expanded as

$$
b=1-2 \frac{\sin ^{2}\left(\frac{r}{2 R_{E}}\right)-\sin ^{2}\left(\frac{\varphi-\varphi_{c}}{2}\right)}{\cos \left(\varphi_{c}\right) \cos (\varphi)} .
$$

Plugging these values into (4), $G$ can be finally computed for any circular footprint of interest.

The simple analytical framework presented is then particularly handy, since it allows to compute the channel load induced by AIS traffic for any given position of the receiver, e.g., for a moving LEO satellite, as well as for different coverage ranges and vessel movement profiles. Within the next section, we will leverage this flexibility to evaluate the achievable performance for different system configurations of interest, with particular attention on the impact of the footprint generated by different flying platforms.

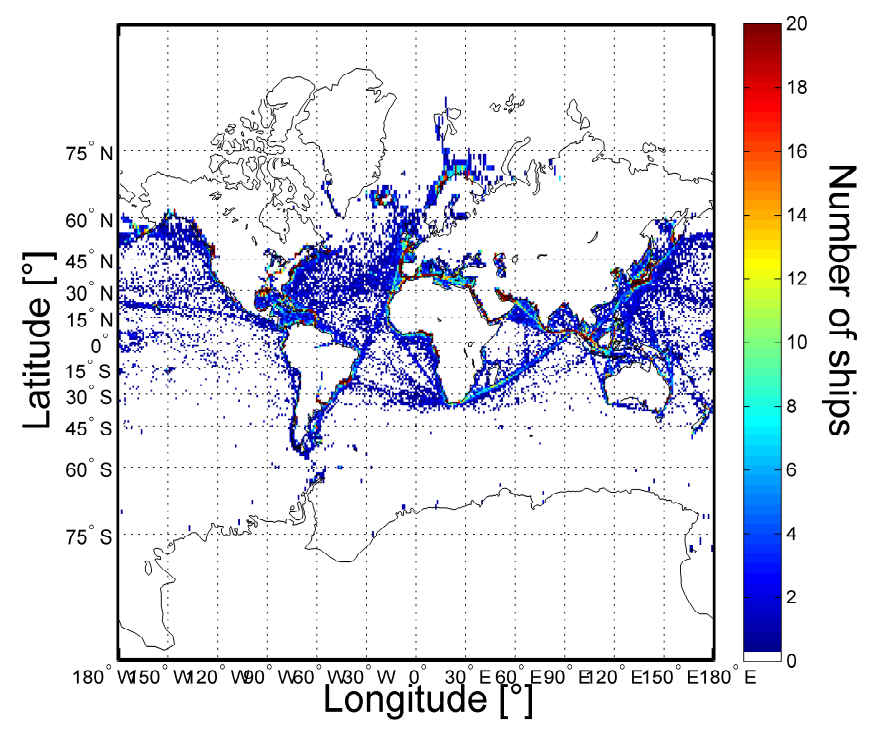

Fig. 1. Ship density from the database generated during the PASTA-MARE project. The ship density is defined as the average number of vessel within a grid cell, based on 10 global satellite AIS scenes. Each global satellite AIS scene retains one position report per vessel within a time frame of 8 days [11]. All grid points where 20 or more ships are present are reported as the maximum scale colour in the figure. There are some heavily ship populated areas, like the Baltic Sea, where the satellite data are not precise due to the very high number of packet collisions experienced by the satellites collecting AIS packets.

\section{COVERAGE RANGe AND AIS RECEPTION PERFORMANCE}

Although satellite detection of AIS messages is very promising for future global vessel and goods tracking, a major and intrinsic impairment to its efficiency is given by the very extended covered footprint, which causes signals from ships very far from each other to possibly overlap at the receiver. In view of the lack of any form of channel coding, packet collisions can in many cases be destructive, inducing poor AIS decoding performance especially in heavily vessel populated regions. Starting from these remarks, in recent years an increasing interest has been drawn by the use of other flying objects for receiving ship-generated information as a complement for satellite-based AIS systems. Relevant results in this direction were presented in [12], where reception at aircraft of AIS messages was proven during flight trials at altitudes of up to $10 \mathrm{~km}$. The concept feasibility was further stressed in [13] with an investigation on the coverage of watered-sea areas by airliners showing how the majority of vessel routes are also covered by airliner. On the other hand, an interesting and complementary approach to reduce the satellite footprint can be represented by advanced antenna design to narrow the generated beam.

While relevant effort is being devoted to the development of such solutions, a clear comparison among them in terms of the critical tradeoff between the larger number of detectable vessels and the lower decoding probability induced by a larger footprint is still missing. From this viewpoint, the framework developed in Section III is particularly useful, as it enables an analytical evaluation of the achievable performance. To this 


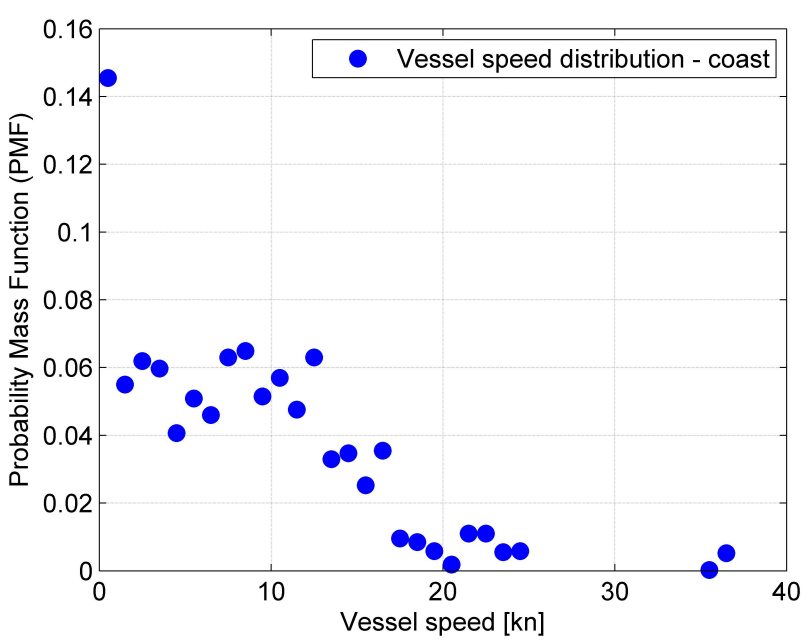

Fig. 2. Vessel speed PMF for ships close to coast. The PMF is derived from the decoded AIS packets received from an aircraft flying at circa $10 \mathrm{~km}$ [12].

aim, three elements need to be fed to the model: a spatial distribution of vessels within the area of interest, a speed profile followed by ships, and a coverage radius offered by the receiver.

As to the first aspect, we resort to the results of the PASTA-MARE project [11], which offers an estimate of the ship position density derived from experimental reception of AIS data at a LEO satellite, as reported in Fig. 1. Real-world data are also employed to describe the speed distribution of vessels. In particular, throughout our analysis we characterize differently the behavior of ships traveling offshore and close to the coast. The former follow a speed profile derived from data gathered from online databases for vessels in open seas or oceans, while the speed of the latter is derived from detected AIS data during the flight trials presented in [12]. In Fig. 2 the probability mass function of ship velocity for vessels close to the coast is presented. The vast majority of them have speeds up to $10 \mathrm{kn}$, as expected due to the proximity to harbours, straits or other constrained areas. The open sea and oceanic ships speed distribution is instead modeled as a Gaussian random variable with mean $12.8 \mathrm{kn}$ and $\sigma=5 \mathrm{kn}$. In Fig. 3 the comparison of the coast vessel speed CDF and the open sea vessel speed CDF is depicted. We can observe that while for the coast vessel speed distribution the large majority of vessel speeds are up to $10 \mathrm{kn}$, for the open sea speed CDF the majority of vessel speeds are up to $15 \mathrm{kn}$ and that below $5 \mathrm{kn}$ very few vessel can be found. Finally, for the characterization of the receiver coverage range, we focus on three cases of interest:

- a common LEO satellite at the altitude of $524 \mathrm{~km}$, which offers a footprint of $2500 \mathrm{~km}$ (1349 Nautical Miles (NM)) in radius;

- a LEO satellite with an enhanced antenna design providing a narrower beam to reduce the incoming AIS load. To this aim, we consider as reference the $D L R$ AISat-1 nanosatellite. The satellite, of approximately $13 \mathrm{~kg}$ in weight is equipped with a $4.2 \mathrm{~m}$ long HighGain helix antenna (see also Fig. 4) and four AIS

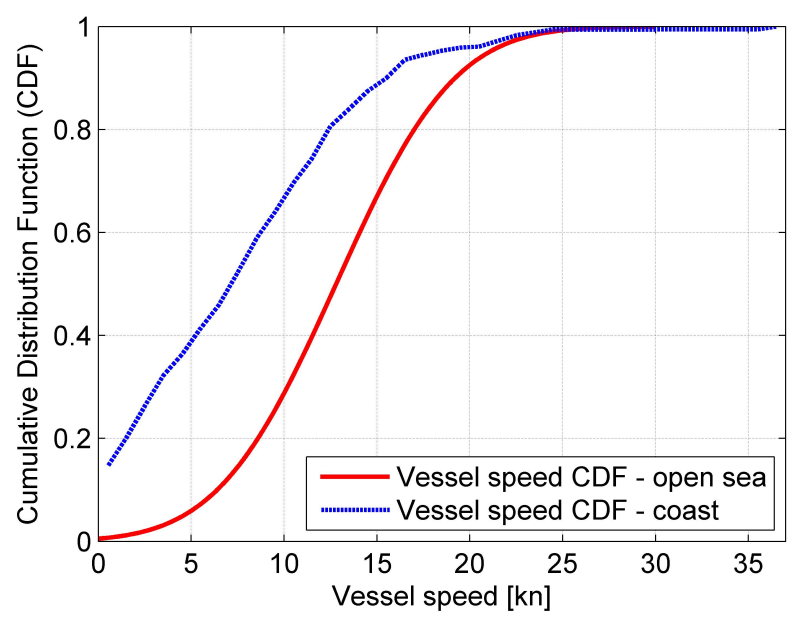

Fig. 3. Vessel CDF of the speed for both ships close to coast and ships in open sea navigation. The coast vessel speed distribution is derived from the AIS packets received during flight trials and elaborated in [12], while the open sea speed distribution is a model derived from 100 ships traveling towards open sea.

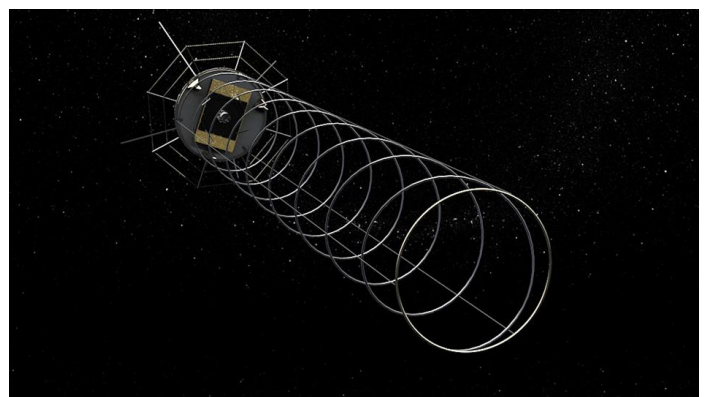

Fig. 4. DLR's AISat-1 with helix antenna.

receivers, developed by the Institute of Space Systems of the German Aerospace Center (DLR) in Bremen. The satellite has an optimized RF-Front End with an HQ filter and 2 VHF dipole antennas. Additional components are a UHF beacon, $1 \mathrm{UHF}$ antenna $(437 \mathrm{MHz})$ and 2 UHF dipole antennas. The AISat- 1 satellite will be launched in 2014 into a sun-synchronized LEO orbit at an altitude of $650 \mathrm{~km}$ on an Indian PSLV from Sriharikota, India, and the resulting offered coverage range is of approximately $350 \mathrm{~km}$.

- an aircraft flying at $10 \mathrm{~km}$. Assuming that all ships with elevation angle $\geq 0^{\circ}$ are received from the aircraft, which has to be shown realistic in [12], the reception radius [13] is in fact $r=\arccos \left(R_{E} /\left(R_{E}+\right.\right.$ $h)) \cdot R_{E} \cong 356 \mathrm{~km}$. By virtue of the very similar footprint with respect to the AISat-1, the two cases will not be distinguished in the following discussion. ${ }^{1}$

Let us then start our discussion focusing on the first

\footnotetext{
${ }^{1}$ Even if offering the same coverage area, the AISat-1 and the airliner-based solutions differ in two main relevant aspects: the altitude (much lower for the airliner, and altering the power distribution profile of incoming AIS messages); and the speed (much higher for the satellite, which covers a certain area for a shorter time). The packet-level framework considered in this paper abstracts these aspects, an investigation of which we leave as part of our future work.
} 


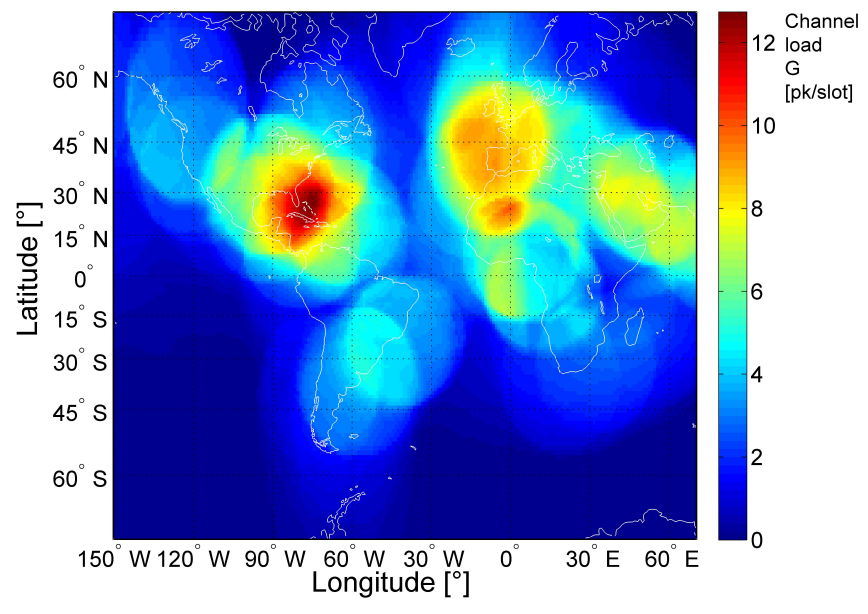

Fig. 5. Channel load $G$ in packets per slot seen at a LEO satellite. Each point in the map represents the channel load seen at the LEO satellite located in that specific longitude and latitude with a reception radius of $2500 \mathrm{~km}$.

scenario. Fig. 5 reports the channel load for a LEO satellite over a wide Earth surface area covering the range $70^{\circ} \mathrm{S}$ to $70^{\circ} \mathrm{N}$ in latitude and $150^{\circ} \mathrm{W}$ to $70^{\circ} \mathrm{E}$ in longitude. Each point on the map represent the channel load for a receiver located towards that latitude and longitude which has a reception radius of $2500 \mathrm{~km}$. We can observe that the maximum channel load at the satellite can exceed 12 packets per slot in the Caribbean area close to Florida and Cuba and can be very high, 8 or more packets per slot, also in other regions like Portuguese and Spanish coasts as well as Mediterranean Sea, Gulf of Guinea and Red Sea. This investigation sheds light on the areas where satellite AIS reception is congested by channel overload and decoding procedures will hardly succeed in detecting packets. Although no physical layer is taken into account in this result, and therefore no advantages due to received power unbalance of the packets for example is considered, the outcome of this study is of interest when designing and operating a satellite in order to identify regions where better (and more complex) detection and decoding techniques are needed.

Moreover, two open questions arise from this investigation: i) how sensible is the channel load variation due to change in the receiver reception range, and ii) what is the best receiver reception radius that maximises the AIS performance. The answers to these two questions are the objective of the reminder of this section. In order to address the first question, we focus on the AIS channel load for a receiver with a reception radius of $356 \mathrm{~km}$ which, as discussed earlier, covers the cases of both AISat-1 and an airliner. The results are presented in Fig. 6. In this case, the focus is on the North America region. We can firstly note that maximum channel load is now limited to less than 2 packets per slot, six times less than in the reference LEO satellite configuration. Moreover, only a limited number of spots close to the coast have a relevant medium channel load and only in the north Mexican Gulf and close to Seattle/Vancouver we exceed 1 packet per slot. Interestingly, the drastic reduction in the reception radius have a huge impact on the channel load. This reduced channel load appears to be much more handleable from the system than the one at the LEO satellite.

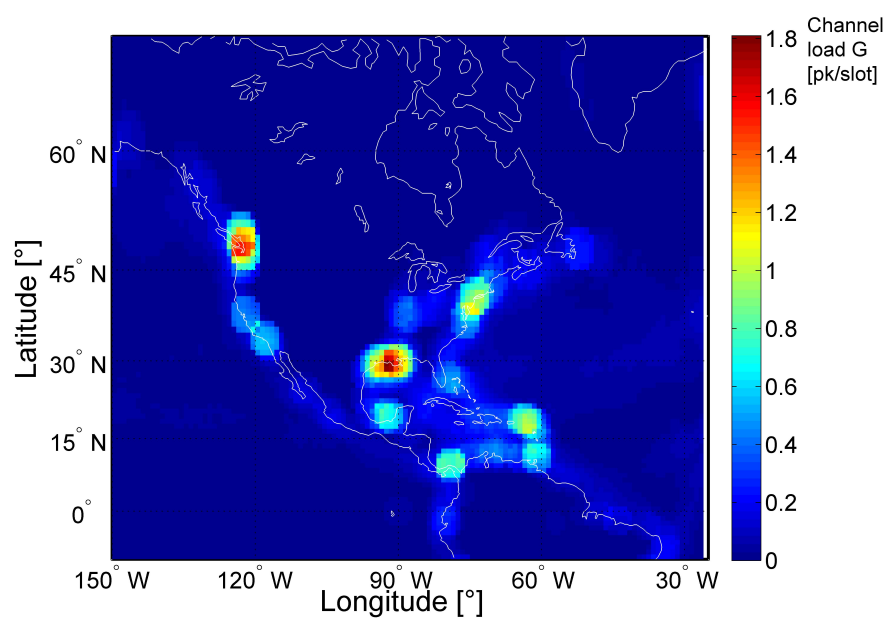

Fig. 6. Channel load $G$ in packets per slot seen at an aircraft flying at 10 $\mathrm{km}$ altitude and assuming that it can receive packets from transmitters with at least $0^{\circ}$ elevation angle and equivalently this is the channel load for the DLR's satellite AISat-1 with the special antenna that reduces the receiver footprint.

As to the second question, we start by elaborating equation (4). The total number $n$ of ships falling within the reception footprint can be derived once the average ship density per square kilometer $d$ is given. In particular, under the assumption of a circular coverage area with radius $r$, the channel load can be written as

$$
G=\pi r^{2} \cdot d \cdot \sum_{i}\left[\omega_{i} \int_{v_{i-1}}^{v_{i}} f_{v}(v) d v\right] .
$$

In order to optimize the system performance, we focus on the throughput, defined as the average number of packets successfully retrieved per slot. Such a metric captures the efficiency of a medium access protocol, and is a direct indicator of how well a flying platform can exploit the available bandwidth. The slotted ALOHA (SA) model employed to describe packets reception prompts the well-known characterization of the throughput in terms $G$ as $S=G e^{-G}$, so that the optimal working point is achieved for unit average channel load ${ }^{2}$. Imposing this condition into (6), the optimum reception radius follows:

$$
r_{o p t}=\sqrt{\frac{1}{\pi \cdot d \cdot \sum_{i}\left[\omega_{i} \int_{v_{i-1}}^{v_{i}} f_{v}(v) d v\right]}} .
$$

The equation prompts how $r_{o p t}$ depends in general, on the specific receiver location and on the vessel speed distribution. The average ship density can be related only with the receiver coverage area or can also be more extended and can cover wider earth surface areas. For example, we can identify an oceanic area and we can perform the radius optimization for that area. In our case we have identified the Atlantic Ocean area delimited by latitudes in the range $\left[15^{\circ} \mathrm{N} ; 45^{\circ} \mathrm{N}\right]$ and longitudes in the range $\left[60^{\circ} \mathrm{W} ; 15^{\circ} \mathrm{W}\right]$, where the optimum radius is found to be $r_{\text {opt }}=1252 \mathrm{~km}$. In order to validate the result of the optimization and to show how this optimization

\footnotetext{
${ }^{2}$ We underline that the AIS standard does not foresee any feedback nor retransmission. Therefore, the system modeled as SA is inherently stable.
} 


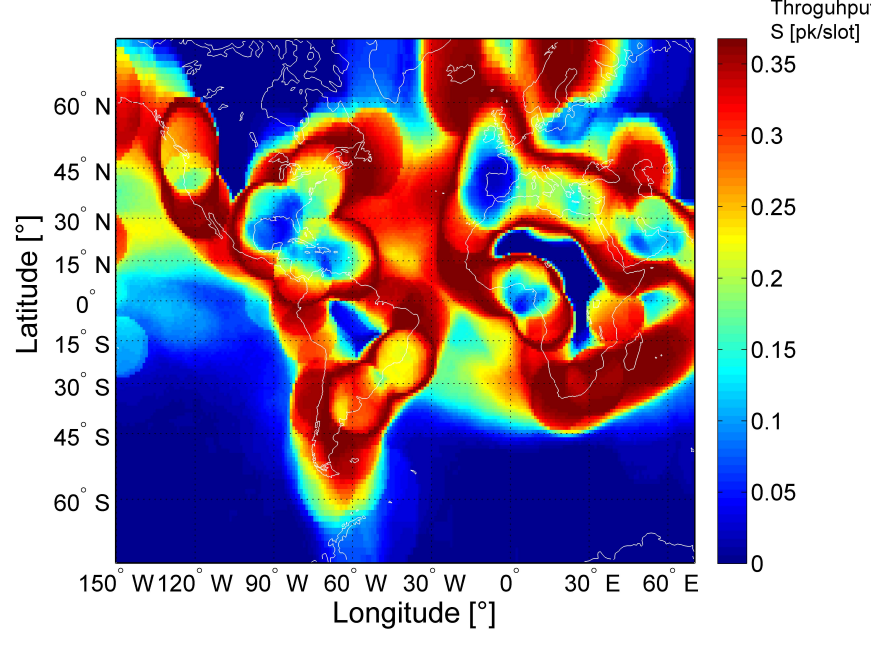

Fig. 7. SOTDMA throughput for a satellite with a reception range radius of $1252 \mathrm{~km}$. This is the radius which optimize the throughput in the north Atlantic Ocean area.

will have an impact on the AIS system performance, we show the throughput results for this reception radius.

In Fig. 7, the throughput results for a satellite with a reception range of $1252 \mathrm{~km}$ in radius is shown. In order to evaluate the throughput, we have computed the channel load as per equation (4), for each Earth surface point. Recalling that the maximum throughput of SA is 0.36 packets per slot, reached for a channel load of 1 packet per slot, we can observe that in the north Atlantic region the throughput is very close to the maximum (above 0.3 packets per slot in the entire area), validating the presented optimization procedure. On the other hand, since the optimization is done exploiting the average ship density in the north Atlantic Ocean the throughput cannot match exactly the maximum in each point because variation in the position dependant ship density with respect to the average can be found. We can also observe that in some medium ship populated regions as the west North America coast or the East South America coast where the channel load in a conventional LEO satellite would have been between 4 and 5 packets per slot (Fig. 5), the throughput with the optimized reception range is very close to the maximum, and therefore the channel load can be deduced to be close to 1 packet per slot. This has a direct advantage in the AIS system performance, because higher throughput can be translated in the better ship tracking.

While throughput is apt to evaluate how efficiently resources are utilized, the primary goal of satellite AIS is to offer worldwide tracking for vessels. Therefore, it is important to also define a metric that captures the capability of detecting ships for a receiver flying over a certain region. To this aim, we introduce the first pass ship detection probability $P_{d e t}$. From this standpoint, it is relevant to observe that one flying platform covers a point on the Earth surface for a certain amount of time, determined by its orbit and speed. In terms of vessel monitoring, then, it is paramount to identify the presence of as many ships as possible within this interval, since the subsequent passage over the same area may take place hours or days after. Thus, we define as detection the event of decoding at the receiver at least one packet sent by a vessel while passing by its position. Let us consider a ship transmitting $k$ AIS messages per frame. Under the medium access model we consider, the probability of detecting such a vessel within one frame is given by $1-\left(1-e^{-G}\right)^{k}$. On the other hand, define as $I(r)$ the number of frames during which the receiver will illuminate the $(\varphi, \lambda)$ location. Regarding transmission patterns as independent across frames, the first pass detection probability can then be expressed as

$$
P_{\text {det }}(\varphi, \lambda, r)=1-\prod_{i=1}^{I(r)}\left(1-e^{G_{i}(\varphi, \lambda, r)}\right)^{k},
$$

where $G_{i}$ is the channel load seen at the receiver during the $i$-th frame.

As discussed, one of the main impairments for satellite AIS is the high packet collision probability especially in highly vessel populated regions. In this way, reducing the reception radius of the satellite, with ad-hoc antenna design as for the AISat-1 of DLR for example, could be of benefit. On the other hand, the reduction of the reception radius of the satellite diminishes the amount of time that the satellite illuminates a certain point on Earth, reducing the packets received (successfully or collided) belonging to a vessel in that particular position. Furthermore, with smaller reception radius, smaller portions of Earth surface are illuminated in each pass and therefore less potential vessels can be tracked. There is in fact, a tradeoff between the reduction of the reception radius and the satellite AIS tracking performances that is well captured by the $P_{\text {det }}$ metric. In particular the channel load $G$ will have a benefit from the footprint shrinking, but on the other hand $I(r)$ will be reduced compared to a common LEO satellite.

In order to make a first analysis of this tradeoff, let us consider a LEO satellite ground track as depicted in Fig. 8, and three reception radiuses for the LEO satellite: i) $r=2500$ $\mathrm{km}$ corresponding to a conventional satellite in Fig. 8(a); ii) $r=1252 \mathrm{~km}$ corresponding to a satellite with the reception radius optimized for oceanic regions in Fig. 8(b); iii) $r=712$ $\mathrm{km}$ corresponding to DLR's AISat-1 satellite Fig. 8(c). The satellite ground track (red line in the figures) and the satellite speed over ground is the same for all the cases. Furthermore, we assume to capture the situation every minute, which corresponds to one AIS frame. The plots in Fig. 8 show the first pass ship detection probability $P_{d e t}$ for the vessels with the lowest packet transmission frequency, which are the vessels sending one AIS packet every $180 \mathrm{~s}$. Such a configuration is of particular interest for two reasons. First, slow-moving vessels are the ones less likely to be detected, so that $P_{\text {det }}$ for them truly represents an indicator of how accurately a receiver can depict the situation on the illuminated area. Secondly, the transmission frequency of 1 message per 180 seconds also characterizes VHF bands recently allocated to the socalled Long Range AIS (LRAIS). These additional channels are foreseen explicitly for satellite reception, and vessels are supposed to distribute AIS packets with the considered frequency regardless of their speed. The reported study, then, also offers hits on the impact of the reception radius on the performance of LRAIS.

Moving back to the plots, in the case of conventional satellite, the Earth surface area covered during one revolution is the highest among all the three cases due to the biggest 


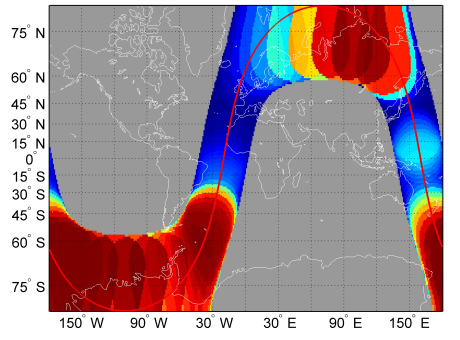

(a) LEO satellite with a reception radius of 2500 $\mathrm{km}$ over its ground track. This represent the reception radius of a standard commercial LEO satellite.

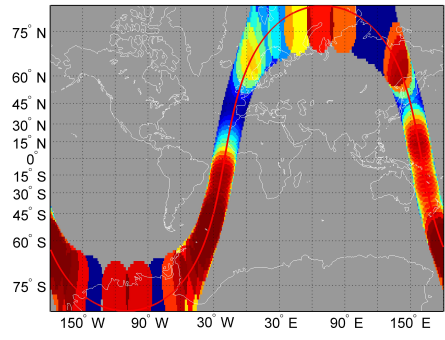

(b) LEO satellite with a reception radius of 1252 $\mathrm{km}$ over its ground track. This reception radius is the one which maximise the throughput for the north Atlantic Ocean.

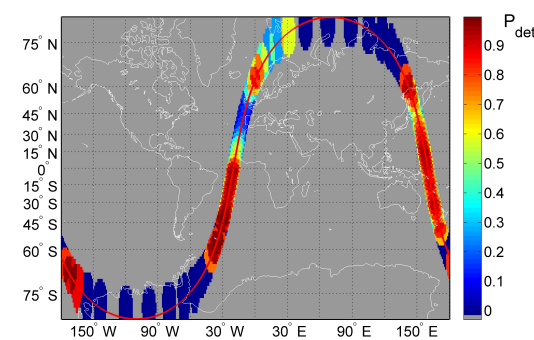

(c) LEO satellite with a reception radius of $712 \mathrm{~km}$ over its ground track. This reception radius is the one of the DLR's AISat-1.

Fig. 8. First pass ship detection probability $P_{\text {det }}$ of ships with the lowest sending rate (1 packet sent every $\left.180 \mathrm{~s}\right)$ for a LEO satellite with different reception radiuses.

reception radius. On the other hand, we can observe that there is a high portion of the ground track where the probability of detection is very low. This is mainly concentrated in two regions of the ground track, on the Atlantic Ocean, close to the African and European coasts and in the Pacific Ocean close to the Japanese and Australian coasts. This result suggests that since these regions are densely populated with vessels, the satellite footprint is too large to provide sufficient tracking capabilities for this class of ships. In other words, the very high channel load is driving the $P_{\text {det }}$ in this case. In the second case in fact, when the reception radius is reduced to half of the conventional satellite, the two regions of the ground track where the $P_{\text {det }}$ is very low are much smaller, and the vast majority of the ground track has a good or acceptable vessel detection probability. It is also interesting to observe that for some satellite positions close to the Arctic and Antarctic regions, $P_{d e t}$ is exactly 0 . This is due to the fact that no ships are received in these regions and shall not be interpreted as a negative result. In the last case, the satellite positions where the $P_{\text {det }}$ is exactly 0 increases, due to the even smaller footprint. On the other hand, in the Pacific Ocean region the first pass vessel detection probability is less sensitive to the high density and also in the Atlantic Ocean region, the locations where the $P_{\text {det }}$ is reduced also w.r.t. the second case.

As a second example, we focus on a small portion of the satellite ground track presented in Fig. 8, the region of the Atlantic Ocean close to the African and European coasts. We focus in this second case to the vessels with the highest packet transmission frequency, which are the vessels sending one AIS packet every 2 s. In Fig. 9 the results of the $P_{\text {det }}$ for this class of vessels is shown for the three reception radius cases. What we can observe is that while for a conventional LEO satellite (red curve) also in the case of highest packet transmission frequency, the $P_{\text {det }}$ drops to less than 0.2 , for the optimized reception radius it is higher than 0.95 while for the DLR's AISat-1 it does not fall below 1 .

\section{COnClusions}

In this work we presented a general but simple and flexible analytical framework for evaluating the channel load of the SOTDMA protocol used in AIS. Exploiting realistic ship distribution over the sea surface and realistic ship speed distribution we evaluated the channel load seen from different flying

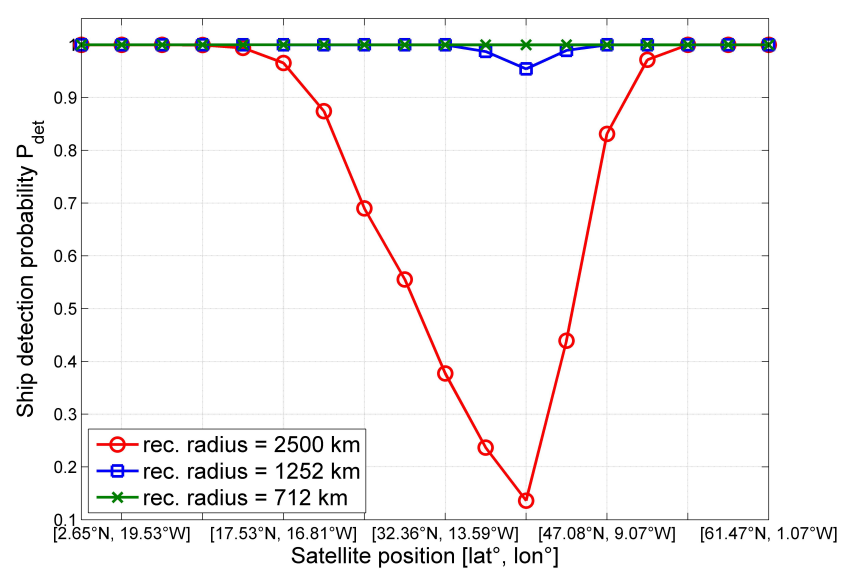

Fig. 9. Ship detection probability $P_{\text {det }}$ of ships with the highest sending rate (1 packet sent every $2 \mathrm{~s}$ ) for a LEO satellite with three different reception radiuses: $2500 \mathrm{~km}, 1252 \mathrm{~km}$ and $712 \mathrm{~km}$. The satellite is passing close to the African and European coasts on the Atlantic Ocean

objects. Common LEO satellites have the largest footprint but suffer from very high channel loads, while aircraft as well as the DLR's AISat-1 due to the smaller footprint appears to be more suitable for AIS data reception in heavily vessel populated regions.

Exploiting the channel load model, an optimization of the reception footprint is also presented in this work, in order to show some possible use cases for the channel load model. The optimized footprint is derived for an oceanic region (Atlantic Ocean) and several results are shown in comparison with the common LEO satellite footprint and DLR's AISat-1 footprint. The ship detection probability for the AIS packet class with the lowest frequency of transmission, is shown to be very high for both the optimized satellite and the DLR's AISat1 assuming a common satellite ground track. On the other hand, the former has the advantage to cover a larger area of the Earth surface, increasing the number of vessels seen in the first pass. Comparison with a common LEO satellite has shown remarkable advantages of the two reduced footprints especially in the densely vessel populated regions. 


\section{ACKNOWLEDGEMENTS}

The research leading to these results has been carried out under the framework of the project "R\&D for the maritime safety and security and corresponding real time services". The project started in January 2013 and is led by the Program Coordination Defence and Security Research within the German Aerospace Center (DLR).

\section{REFERENCES}

[1] Recommendation ITU-R M.2169, "Improved satellite detection of AIS," ITU, Tech. Rep., 2009.

[2] Recommendation ITU-R M.1371-4, "Technical characteristics for an automatic identification system using time-division multiple access in the VHF maritime mobile band," ITU, Tech. Rep. Recommendation ITU-R M.1371-4, 042010.

[3] T. Eriksen, G. Hoye, B. Narheim, and B. Jenslokken Meland, "Maritime traffic monitoring using a space-based AIS receiver," Acta Astronautica, vol. 58, pp. 537-549, 2006.

[4] T. Eriksen, A. N. Skauen, B. Narheim, O. Helleren, O. Olsen, and R. B. Olsen, "Tracking Ship Traffic with Space-Based AIS: Experience Gained in First Months of Operations," in Waterside Security Conference (WSS), 2010 International, Carrara, Italy, 3-5 November 2010, pp. $1-8$.

[5] J. A. Larsen, H. P. Mortensen, and J. D. Nielsen, "An SDR based AIS Receiver for Satellites," in Recent Advances in Space Technologies (RAST), 2011 5th International Conference on, Istanbul, Turkey, 9-11 June 2011, pp. 526-531.

[6] M. A. Cervera, A. Ginesi, and K. Eckstein, "Satellite-based vessel Automatic Identification System: A feasibility and Performance analysis," Int. J. Satell. Commun. Network, vol. 29, Issue 2, pp. 117-142, March/April 2011.

[7] J. A. Larsen and H. P. Mortensen, "In Orbit Validation of the AAUSAT3 SDR based AIS receiver," in Recent Advance in Space Technologies (RAST), 2013 6th International Conference on, Istanbul, Turkey, 12-14 June 2013, pp. 487-491.

[8] Electronic Communications Committee, "Information Paper on VHF Data Exchange System (VDES), CPG PTC(13) INFO 16," CEPT, Tech. Rep., 2013.

[9] F. Clazzer, A. Munari, M. Berioli, and F. Lazaro Blasco, "On the Characterization of AIS Traffic at the Satellite," in MTS/IEEE Oceans '14, Taipei, Taiwan, 2014.

[10] P. Burzigotti, A. Ginesi, and G. Colavolpe, "Advanced receiver design for satellite-based automatic identification system signal detection," Int. J. Satell. Commun. Network, vol. 30, Issue 2, pp. 52-63, March/April 2012.

[11] (2011) Maritime traffic density - results of PASTA MARE project. [Online]. Available: https://webgate.ec.europa.eu/maritimeforum/content/1603

[12] S. Plass, R. Poehlmann, A. Dammann, and C. Gentner, "Investigations on AIS Signal Reception on Aircraft at Higher Altitudes," in MTS/IEEE Oceans '14, Taipei, Taiwan, 2014.

[13] S. Plass and R. Hermenier, "Study on Worldwide Dectection of AIS Signals via Airliners," in MTS/IEEE Oceans '14, Taipei, Taiwan, 2014. 